\title{
Design and implementation of flexible laboratory system for beam propagation study through weak atmospheric turbulence
}

\author{
Carolina Rickenstorff *, José A. Rodrigo and Tatiana Alieva \\ Universidad Complutense de Madrid, Facultad de Ciencias Físicas, Ciudad Universitaria s/n, Madrid 28040, \\ Spain.
}

\begin{abstract}
Different applications such as astronomy, remote optical sensing and free space optical communications, among others, require both numerical and laboratory experimental simulations of beam propagation through turbulent atmosphere prior to an outdoor test. While rotating phase plates or hot chambers can be applied to such studies, they do not allow changing the atmospheric conditions and the propagation distance in situ. In contrast, the spatial light modulators (SLMs) are a flexible alternative for experimental turbulence simulation. In this work we consider an experimental setup comprising two SLMs for studying laser beam propagation in weak atmospheric turbulence. The changes of atmospheric conditions and propagation distances are properly achieved by the adjustment of the phase screens and the focal distances of digital lenses implemented in both SLMs. The proposed system can be completely automatized and all its elements are in fixed positions avoiding mechanical misalignment. Its design, propagation distance and atmospheric condition adjustment are provided. The setup performance is verified by numerical simulation of Gaussian beam propagation in the weak turbulence regime. The obtained parameters: scintillation index, beam wander and spreading are compared to their theoretical counterparts for different propagation distances and atmospheric conditions.
\end{abstract}

Keywords: Atmospheric turbulence simulator, Propagation through random medium, Opto-electronic system design.

\section{INTRODUCTION}

Light beam distortion by atmospheric turbulence is an important issue for different applications such as astronomy, remote optical sensing and free space optical communications (FSOC), among others. These applications require both numerical and indoor experimental simulations of beam propagation through turbulent atmosphere prior to an outdoor test. Here we consider a programmable laboratory setup based on two spatial light modulators (SLMs) for beam propagation study through weak atmospheric turbulence.

The atmospheric turbulence is caused by a series of air whirls of decaying size originated by temperature gradients. Such process can be statistically modeled by the Von Karman power spectrum density

$$
\Phi(\kappa)=0.023 r_{0}^{-5 / 3}\left(\kappa^{2}+\kappa_{0}^{2}\right)^{-11 / 6} \exp \left(-\kappa^{2} / \kappa_{m}^{2}\right),
$$

where $r_{0}$ is the Fried parameter related to the coherence length of the transmitted field while $\kappa_{0}$ and $\kappa_{m}$ are the spatial frequencies corresponding to the maximum and minimum whirl sizes ranging from $1 \mathrm{~cm}$ to tenths of meters. The turbulence's intensity in the atmosphere is measured by the structure parameter $C_{n}^{2}$ whose typical values belong to the interval $10^{-17}-10^{-12} \mathrm{~m}^{-2 / 3}$, being the latter value related to strong fluctuations. During the propagation in the atmosphere an optical beam is degraded affecting its transmission information capacity. There are three parameters usually employed for characterization of beam distortion: Scintillation index (SI), beam wander and beam spreading. The SI is defined as:

\footnotetext{
${ }^{*}$ E-mail: obice4@yahoo.com.mx
} 


$$
\sigma_{I}^{2}=\frac{\left\langle I^{2}\right\rangle}{\langle I\rangle^{2}}-1
$$

where $\langle\cdot\rangle$ stands for ensemble average, describes the intensity $(I)$ variance, while the beam wander corresponds to the beam's centroid variance in the receiver plane and the beam spreading is the widening of the beam average profile as it traverses the atmosphere in addition to normal diffraction. In many applications it is enough to consider the weak turbulent regime where the dimensionless parameter $\sigma_{R}^{2}$ known as Rytov variance fulfills the condition: $\sigma_{R}^{2}=1.23 k^{7 / 6} C_{n}^{2} L^{11 / 6}<1$. Here $k=2 \pi / \lambda$ is the wave number, $\lambda$ is the wavelenght and $L$ is the propagation path. Under this model several simplifications can be done and tractable mathematical expressions for SI, beam wander and beam spreading in the case of Gaussian beams exist. ${ }^{1}$ Due to the lack of rigorous theory describing the propagation of other beam types of current interest in information transmission (LaguerreGaussian, Bessel, Airy beams, etc) it can be assumed that a good agreement between theoretically predicted and experimentally observed propagation of Gaussian beams through the atmosphere simulator supports the correct functioning of the proposed setup for other beams. Then we consider that the beam at the input plane of the simulator is monochromatic and linearly polarized Gaussian beam with complex field amplitude

$$
U_{0}(r, 0)=A_{0} \exp \left(-\frac{r^{2}}{W_{0}^{2}}-i \frac{k r^{2}}{2 F_{0}}\right),
$$

where $W_{0}$ and $F_{0}$ are the initial beam radius and curvature radius, respectively.

\section{SYSTEM CONFIGURATION}

In the weak turbulent regime, the atmosphere can be simulated by a number of thin random phase screens located in certain positions along the optical path. ${ }^{2}$ Following this approach, an horizontal atmospheric path length $L$ characterized by the Fried parameter $r_{0}=\left(0.4229 k^{2} C_{n}^{2} L\right)^{-3 / 5}$ can be properly represented ${ }^{3}$ by two phase screens described by the parameters $r_{01}$ and $r_{02}$ obtained from the equations

$$
\begin{aligned}
r_{0}^{-5 / 3} & =r_{01}^{-5 / 3}+r_{02}^{-5 / 3}, \\
\frac{\sigma_{R}^{2} k^{5 / 6}}{5.32} & =r_{01}^{-5 / 3}\left(z_{2}+z_{3}\right)^{5 / 6}+r_{02}^{-5 / 3} z_{3}^{5 / 6},
\end{aligned}
$$

where the separation from the input plane to the first phase screen is $z_{1}$, the separation between the screens is $z_{2}$, and the distance from the second screen to the output plane is $z_{3}$ such that $z_{1}+z_{2}+z_{3}=L$. Solving the system of equations the real positive values of the $r_{01}$ and $r_{02}$ are found for the relation between the distances: $z_{3}<0.4845 L<z_{2}+z_{3}$. For the laboratory implementation of the simulator the distances $z,(i=1,2,3)$ have to be re-scaled. Using the $A B C D$ matrix formalism it has been shown ${ }^{4}$ that they can be replaced by the intervals $z_{i}^{\prime}\left(\sum z_{i}^{\prime}=L^{\prime}\right.$ is the laboratory propagation path) each one embedded into two spherical lenses where the beam of radius $W_{0}^{\prime}$ propagates instead of the free space (non-scaled) beam of radius $W_{0}$. Applying this procedure the scaling parameters $\alpha_{j}$ and focal distances $f_{j}$ of the lenses in the laboratory simulator have to satisfy to the following system of equations:

$$
\begin{aligned}
\alpha_{0} & =W_{0}^{\prime} / W_{0}, \\
\alpha_{1} & =z_{1}^{\prime} /\left(\alpha_{0} z_{1}\right)=W_{1}^{\prime} / W_{1}=r_{01}^{\prime} / r_{01}, \\
\alpha_{2} & =z_{1} z_{2}^{\prime} \alpha_{0} /\left(z_{1}^{\prime} z_{2}\right)=W_{2}^{\prime} / W_{2}=r_{02}^{\prime} / r_{02}, \\
\alpha_{3} & =z_{1}^{\prime} z_{2} z_{3}^{\prime} /\left(\alpha_{0} z_{1} z_{2}^{\prime} z_{3}\right)=W_{3}^{\prime} / W_{3}=W^{\prime} / W, \\
f_{0} & =z_{1}^{\prime} \alpha_{0} /\left(\alpha_{0}-\alpha_{1}\right), \\
f_{1} & =z_{1}^{\prime} z_{2}^{\prime} \alpha_{1} /\left[z_{1}^{\prime}\left(\alpha_{1}-\alpha_{2}\right)+z_{2}^{\prime}\left(\alpha_{1}-\alpha_{0}\right)\right], \\
f_{2} & =z_{2}^{\prime} z_{3}^{\prime} \alpha_{2} /\left[z_{2}^{\prime}\left(\alpha_{2}-\alpha_{3}\right)+z_{3}^{\prime}\left(\alpha_{2}-\alpha_{1}\right)\right], \\
f_{3} & =z_{3}^{\prime} \alpha_{3} /\left(\alpha_{3}-\alpha_{2}\right),
\end{aligned}
$$


where subindex $j$ stands for the input plane $(j=0)$, the first phase screen plane $(j=1)$, the second phase screen plane $(j=2)$, and the output plane $(j=3)$. The transverse scaling parameters $\alpha_{j}=W_{j}^{\prime} / W_{j}$ are defined as the ratio between laboratory $W_{j}^{\prime}$ and free space $W_{j}$ beam radii at the corresponding plane. If the outdoor beam has a curvature radius $F_{0}$, then the curvature radius of the laboratory beam is given by $F_{0}^{\prime}=F_{0} \alpha_{0}^{2}$. The figure (1) shows a generic configuration of the proposed setup considering two reflective SLMs: SLM-1 and SLM-2, for the phase screens implementation with re-scaled values $r_{0 j}^{\prime}=\alpha_{j} r_{0 j}$. The lenses $\mathcal{L}_{1}$ and $\mathcal{L}_{2}$ (with focal distances $f_{1}$ and $f_{2}$, respectively) are digitally encoded together with the phase screens into their respective SLMs. In order to make the setup completely electronically controlled the entrance lens $\mathcal{L}_{0}$ (focal distance $f_{0}$ ) has to be a fluidic lens or being digitally encoded into an additional SLM. In the latter case the implementation of this digital lens can be combined with holographic generation of the input beam of various shapes. The lens $\mathcal{L}_{3}$ with the focal distance $f_{3}$ is ignored since only intensity measurements of the transmitted field are performed. The application of digital lenses brings important benefits: the variation of the simulated path through the turbulent atmosphere $L$ is achieved by a programmable way without changing the positions of the elements of the setup.

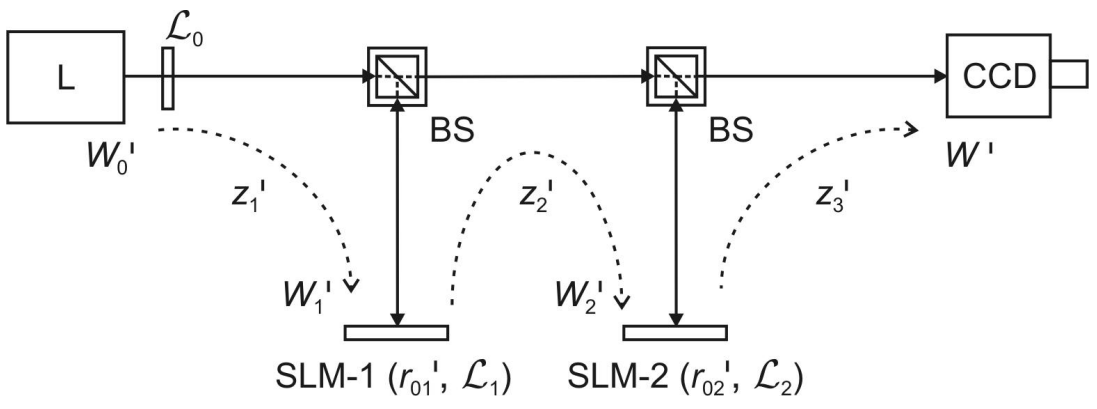

Fig. 1. Experimental configuration of the turbulent atmosphere simulator. L: laser, BS: beam splitter, SLM: spatial light modulator.

Although the Eqs.(5) allow different solutions which can be used to vary $L$ without changing $\alpha_{0}$ and $z_{i}^{\prime}$, in order to simplify the analysis we consider equidistant distances $z_{i}^{\prime}=z^{\prime}$ and $z_{i}=z$ in the laboratory and distretized free space atmosphere simulators. In this case $z_{i}^{\prime} / z_{i}=\gamma, L^{\prime}=\gamma L, \alpha_{0}=\alpha_{2}, \alpha_{1}=\alpha_{3}=\gamma / \alpha_{0}$, and

$$
f_{0}=-\frac{2 f_{1} \alpha_{0}^{2}}{\gamma}=2 f_{2}=\frac{z^{\prime} \alpha_{0}^{2}}{\alpha_{0}^{2}-\gamma}
$$

The characteristics of the SLMs and digital camera (window and pixel sizes, dynamic range, etc) restrict the values of $\alpha_{0}, \gamma, f_{j}$ and the parameter $r_{0, j}$ which can be used. Indeed, the beam radius in SLM-1, SLM-2 and the detector have to match to their active areas and not being too small for proper sampling. Taking into account the beam wander effects, it is reasonable to assume that a device window has to be twice as large than the effective diameter of the beam arriving at its plane if turbulence effects are ignored. This issue can be checked by considering the propagation of a Gaussian beam with input parameters $W_{0}^{\prime}$ and $F_{0}^{\prime}$ arriving to each device plane. To illustrate this analysis we consider a collimated Gaussian beam $\left(F_{0}^{\prime}=\infty, \lambda=532 \mathrm{~nm}, W_{0}^{\prime}=0.5 \mathrm{~mm}\right)$ and the appropriate laboratory distances $z_{i}^{\prime}=z^{\prime}=0.5 \mathrm{~m}$. If we choose $\alpha_{0}=0.012$, the real world beam radius that is simulated is $W_{0}=W_{0}^{\prime} / \alpha_{0}=41.6 \mathrm{~mm}$. In Fig. 2 the beam radii at the planes of the SLMs $\left(W_{1,2}^{\prime}\right)$ and the detector $\left(W^{\prime}\right)$ are calculated for the simulated propagation distance $L \in[1 \mathrm{~km}, 20 \mathrm{~km}]$. We underline that for distances below $2 \mathrm{~km}$ the size of the detector and SLM-2 displays has to be significantly large in order to host the beam, while for large distances $L>10 \mathrm{~km}$ the spatial resolution of these devices is important due to small beam radius. Note that the beam radius $W^{\prime}$ at the detector plane scales down with the simulated propagation distance $L$ despite that the input beam is collimated. This might be counter-intuitive but this fact is explained by the inverse dependence between $\alpha_{3}$ and $L$. 


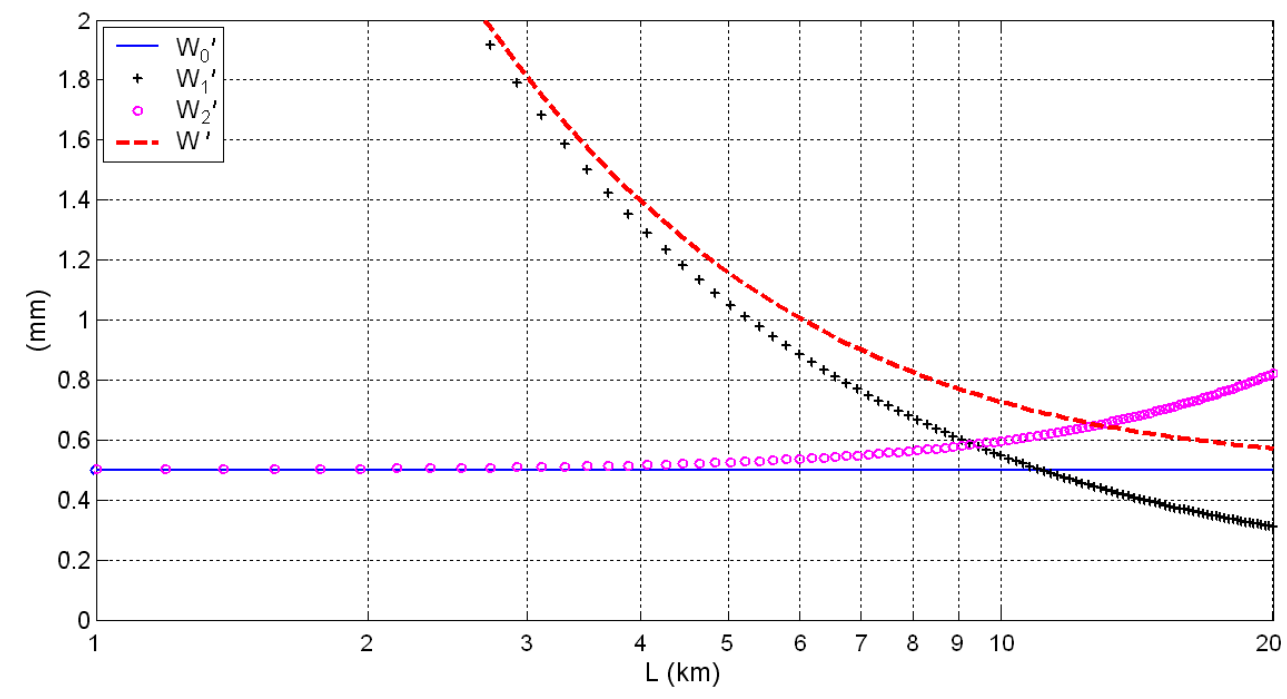

Fig. 2. Gaussian beam radii at the planes corresponding to emitter $W_{0}^{\prime}$, SLM-1 $W_{1}^{\prime}$, SLM-2 $W_{2}^{\prime}$, and detector $W^{\prime}$ versus the propagation distance $L$ for the setup parameters $\lambda=532 \mathrm{~nm}, z^{\prime}=0.5 \mathrm{~m}, W_{0}^{\prime}=0.5 \mathrm{~mm}, F_{0}^{\prime}=\infty$ and $\alpha_{0}=0.012$.

The spatial resolution of the SLMs is also a very important issue for digital lens and phase screen encoding. In Fig.3 the focal distances of the required digital lenses as a function of $L$ are shown. We underline that no lenses are needed to simulate the propagation at the distance $L=10.4 \mathrm{~km}$. At this point the focal distances go to infinity and then reverse signs. The actually available varifocal lenses, proposed for the implementation of the $\mathcal{L}_{0}$, allows focal distance variation in the range $\left|f_{0}\right| \in[0.1 \mathrm{~m}, 1 \mathrm{~m}]$ as cited elsewhere, ${ }^{5}$ that reduces the propagation interval to $L \in[3 \mathrm{~km}, 7 \mathrm{~km}]$. The pixel size of the commonly used SLMs based on the liquid crystal technology $(8-20 \mu \mathrm{m})$ have similar limitations for lens encoding.

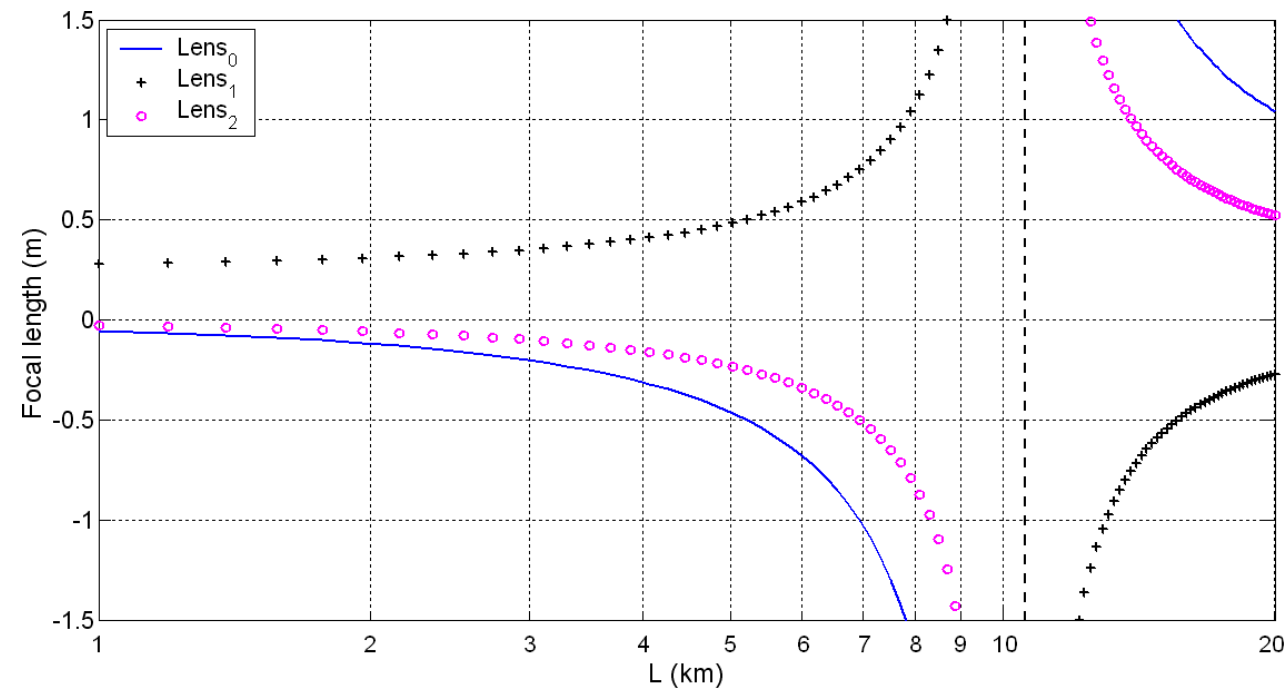

Fig. 3. Focal lengths of the digital lenses required for simulation of the beam propagation at distance $L$ for the setup parameters $\lambda=532 \mathrm{~nm}, z^{\prime}=0.5 \mathrm{~m}, W_{0}^{\prime}=0.5 \mathrm{~mm}, F_{0}^{\prime}=\infty$ and $\alpha_{0}=0.012$.

For correct phase screen representation by the SLM as a rule of thumb one might require that the encoded value of the Fried parameter $r_{0 j}$ has sufficient pixel number $10 \times s<r_{0 j}^{\prime}=\alpha_{j} r_{0 j}<100 \times s$, where $s$ is the SLM pixel size. This fact may reduce the range of the atmospheric turbulence conditions which can be simulated by a given setup. 
Note that using the same devices the limitations of the propagation distance and the turbulence strength can be modified without any physical displacement using the general system of equations Eqs.(5). We still consider equidistant laboratory positions $z_{i}^{\prime}=z^{\prime}$ while the position of the free space phase screens $z_{i}$ is changed yielding proper combination of the scaling coefficients, focal distances and Fried parameters in the atmosphere model.

\section{NUMERICAL SIMULATIONS}

The performance of the turbulence simulator mimicking the behavior of a collimated Gaussian beam propagating in weak turbulence was evaluated using Matlab routines. We choose the following setup parameters $\lambda=532 \mathrm{~nm}$, $z_{i}=z, z_{i}^{\prime}=z^{\prime}=0.5 \mathrm{~m}, W_{0}^{\prime}=0.5 \mathrm{~mm}, \alpha_{0}=0.012$ and SLMs pixel size $19 \mu \mathrm{m}$ already mentioned in section 2. It was first verified that the digital lens implementation was correct comparing the width of the beam propagated through the system without turbulence $W^{\prime}$ with the expected theoretical value $W_{\text {Theo }}^{\prime}=\alpha_{3} W_{d}$, where $W_{d}=W_{0} \sqrt{1+\Lambda_{0}^{2}}$ is the beam spreading due to diffraction in free space and $\Lambda_{0}=2 L /\left(k W_{0}^{2}\right)$. The output amplitude profile (the square root of the beam intensity) was calculated averaging the profile pair obtained from sweeping the $x-y$ axes of the 2D image and applying a numerical fit to the data to determine more accurately the beam radius. The plots in Fig. 4 show that the beam profiles at the setup output plane simulated for the propagation distances $L=[1,5,10.4,20] \mathrm{km}$ fit with the theoretically predicted ones. This demonstrates the correct optical scaling of the setup and sufficient digital lens sampling. However, we observe that the size of camera sensor (here it is $10 \mathrm{mmx} 10 \mathrm{~mm}$ ) is not appropriate for the beam propagation simulation at the distance of $L=1 \mathrm{~km}$.
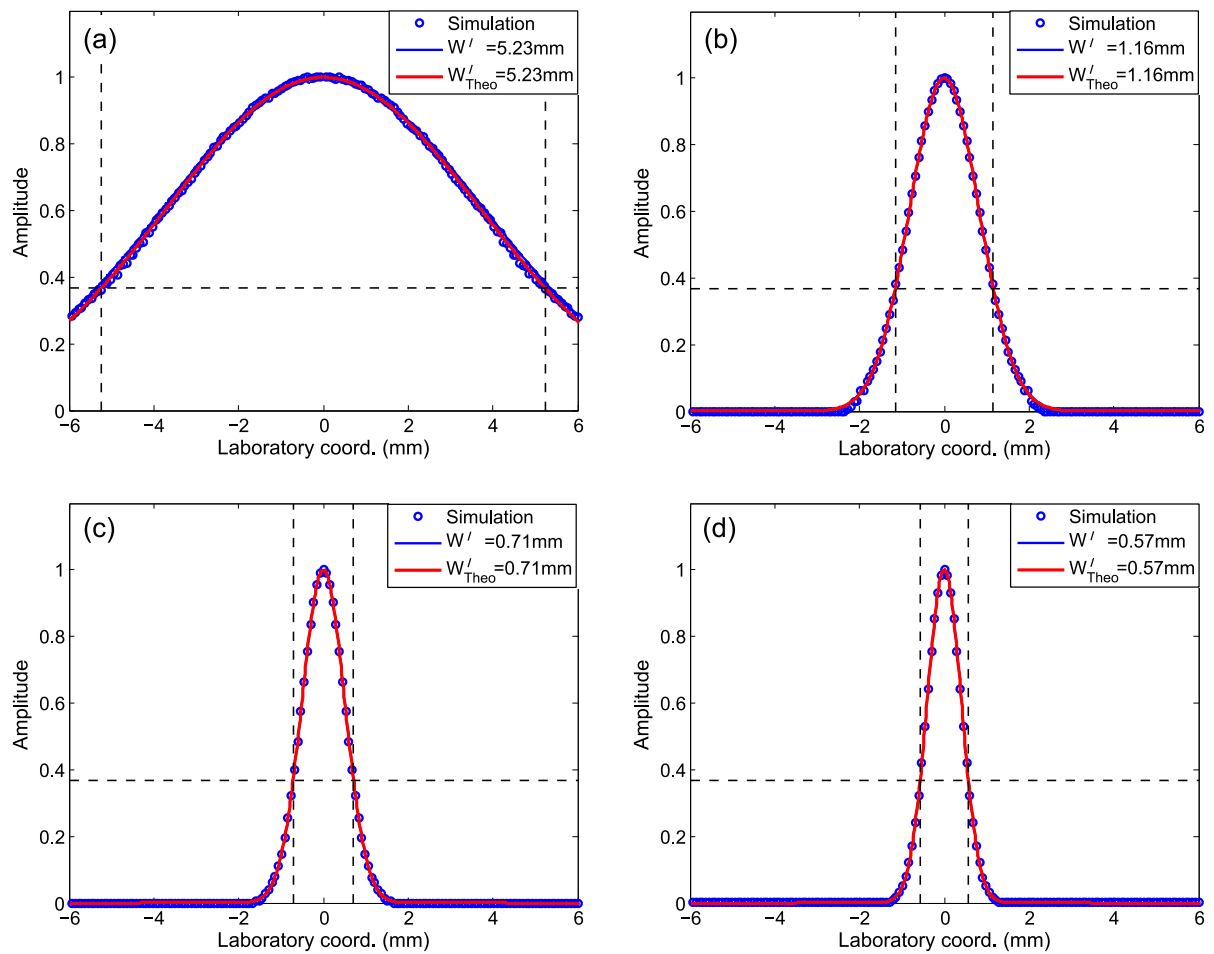

Fig. 4. Beam amplitude profile at the output plane of the simulator $\left(W_{0}^{\prime}=0.5 \mathrm{~mm}\right)$ corresponding to the free space propagation at the distance: (a) $L=1 \mathrm{~km},(\mathrm{~b}) L=5 \mathrm{~km},(\mathrm{c}) L=10.4 \mathrm{~km}$ and (d) $L=20 \mathrm{~km}$.

The phase screens simulating the beam propagation at the distances $L \in[4-6] \mathrm{km}$ through weak turbulent atmosphere: $C_{n}^{2}=[0.5-2] \times 10^{-16} \mathrm{~m}^{-2 / 3}$ have been encoded together with the corresponding digital lenses in the SLMs. To avoid scintillation leakage in the inter-screen separation ${ }^{26}$ we considered the more stringent limitation for weak turbulence conditions $\sigma_{R}^{2} \leq 0.3$. An ensemble (100 pairs) of random phase $512 \times 512$ pixel screens for each atmospheric condition and distance $L$ has been generated following the sub-harmonic addition method. ${ }^{2}$ The typical phase screens for $L=6 \mathrm{~km}$ and different parameters $C_{n}^{2}$ displayed in Fig.5. 

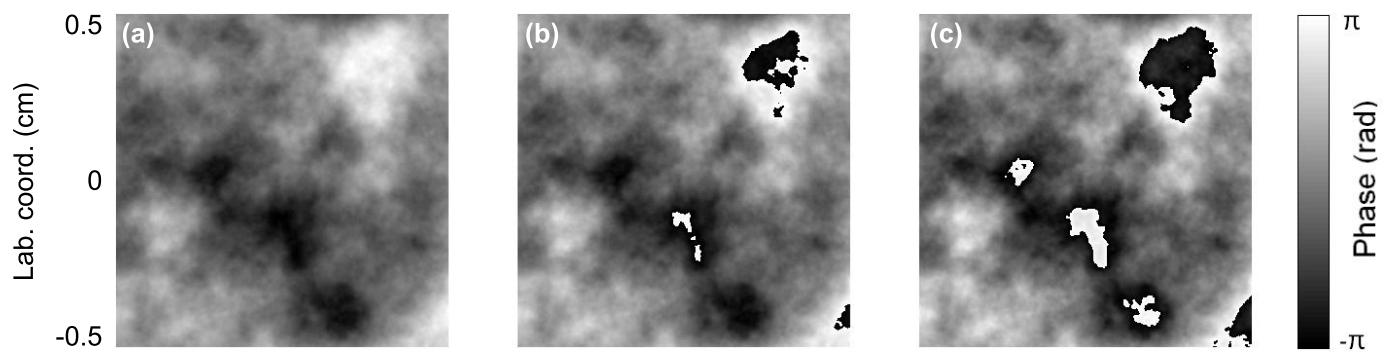

Fig. 5. Left to right: Qualitative examples of atmospheric phase screens with increasing turbulent strength $r_{02}^{\prime}$ for the setup parameters $\lambda=532 \mathrm{~nm}, z^{\prime}=0.5 \mathrm{~m}, W_{0}^{\prime}=0.5 \mathrm{~mm}, F_{0}^{\prime}=\infty, \alpha_{0}=0.012$ and propagation distance $L=6 \mathrm{~km}$. (a) $C_{n}^{2}=1 \times 10^{-16} \mathrm{~m}^{-2 / 3}$, (b) $C_{n}^{2}=1.5 \times 10^{-16} \mathrm{~m}^{-2 / 3}$, (c) $C_{n}^{2}=2 \times 10^{-16} \mathrm{~m}^{-2 / 3}$.

From a total of 100 beam realizations such as the ones shown in Fig.6(a)-(c) the beam characteristics: spreading, wander and scintillation were calculated and compared in Fig.7 with the theoretical expressions derived in Ref.[1]. The beam spreading - beam radius $W$ obtained from the profile of the averaged over 100 realizations intensity distribution (as the one shown in Fig.6(d))- was compared with the theoretical expression

$$
W_{\text {Theo }}=W_{d} \sqrt{1+1.33 \sigma_{R}^{2} \Lambda^{5 / 6}},
$$

where $\Lambda_{0}=2 L /\left(k W_{0}^{2}\right)$ and $\Lambda=\Lambda_{0} /\left(1+\Lambda_{0}^{2}\right)$. The results for various atmospheric conditions and distances are shown in the Fig.7(a). Except for the non turbulent case, the simulated beam spreading is smaller than expected.
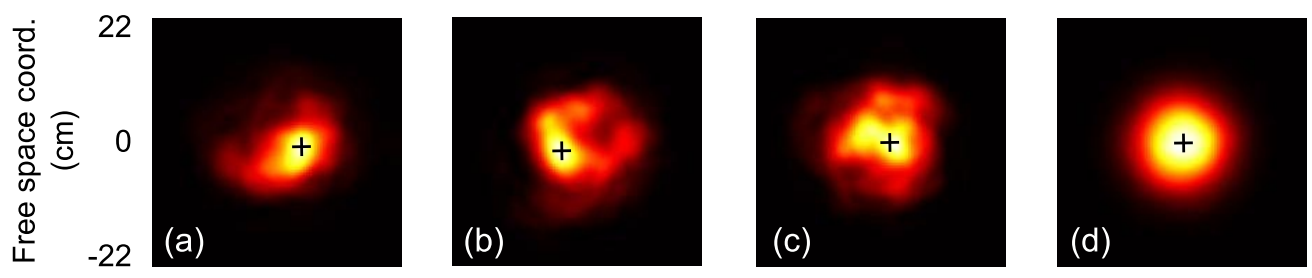

Fig. 6. (a)-(c) Realizations of the transmitted beam (input radius $W_{0}=0.5 \mathrm{~mm} / \alpha_{0}=41.6 \mathrm{~mm}$ ) corresponding to $L=5 \mathrm{~km}$ and $C_{n}^{2}=2 \times 10^{-16} \mathrm{~m}^{-2 / 3}$. (d) Beam intensity distribution averaged over 100 realizations. The black cross indicates the image's centroid.

The beam wander $\sqrt{\left\langle r_{c}^{2}\right\rangle}$ was calculated from the ensemble of 100 centroid values. For each image realization the centroid indicated by the black cross in Fig.6(a)-(c) is given by:

$$
\mathbf{r}_{c}=\left(x_{c}, y_{c}\right)=\left\{M_{10} / M_{00}, M_{01} / M_{00}\right\},
$$

with $M_{i j}=\sum_{x} \sum_{y}(x-\bar{x})^{i}(y-\bar{y})^{j} I(x, y)$ being the image's moment, where $I(x, y)$ the intensity distribution and $(\bar{x}, \bar{y})$ is the centroid of the image averaged over the ensemble, see Fig.6(d). The obtained beam wander after re-scaling (multiplication by $1 / \alpha_{3}$ ) is compared in Fig.7(b) with the theoretical value calculated from

$$
<r_{c}^{2}>=2.42 C_{n}^{2} L^{3} W_{0}^{-1 / 3}\left[1-\left(\frac{\kappa_{0}^{2} W_{0}^{2}}{1+\kappa_{0}^{2} W_{0}^{2}}\right)^{1 / 6}\right] .
$$

We conlude that the simulated beam wander is half of the theoretical counterpart. This result as well the lower beam spreading can be explained by the lack of low frequency content of the phase screen generation method stated by other authors. ${ }^{7}$

Finally, using the formula (2) the SI measured at the center of the averaged image (on axis) was compared with the theoretical value in Fig.7(c). The theoretical expression for the on axis SI of the collimated Gaussian beam corresponding to the Von Kàrman turbulence model is given by 


$$
\begin{aligned}
\sigma_{I}^{2}(L)= & 3.86 \sigma_{R}^{2}\left\{0.4 \frac{\left[(1+2 \Theta)^{2}+\left(2 \Lambda+3 / Q_{m}\right)^{2}\right]^{11 / 12}}{\left[(1+2 \Theta)^{2}+4 \Lambda^{2}\right]^{1 / 2}} \sin \left(\frac{11}{6} \varphi_{1}+\varphi_{2}\right)\right. \\
& \left.-\frac{6 \Lambda}{Q_{m}^{11 / 6}\left[(1+2 \Theta)^{2}+4 \Lambda^{2}\right]}-\frac{11}{6}\left(\frac{1+0.31 \Lambda Q_{m}}{Q_{m}}\right)^{5 / 6}\right\},
\end{aligned}
$$

where $Q_{m}=L \kappa_{m}^{2} / k, Q_{0}=L \kappa_{0}^{2} / k, \Theta=1 /\left(1+\Lambda_{0}^{2}\right)$ and

$$
\varphi_{1}=\arctan \left[\frac{(1+2 \Theta) Q_{m}}{3+2 \Lambda Q_{m}}\right], \quad \varphi_{2}=\arctan \left[\frac{2 \Lambda}{1+2 \Theta}\right] .
$$

A good agreement between the simulated and the theoretical scintillation values depicted in the Fig.7(c) demonstrates that this characteristic is correctly represented by the phase screens.
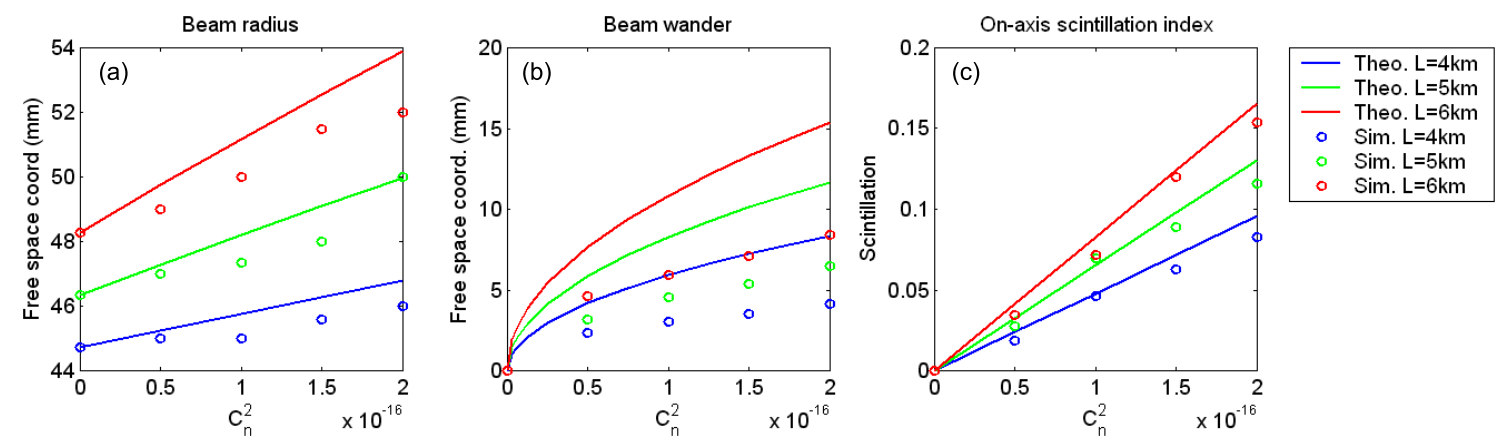

Fig. 7. (Colour online) Theoretical (line) and simulated (circles) plots corresponding to beam radius (a), beam wander (b) and on axis $r=0$ scintillation index (c) for the collimated Gaussian beam propagated at distances $L=4 \mathrm{~km}$ (blue), $L=5 \mathrm{~km}$ (green), $L=6 \mathrm{~km}$ (red), as a function of the structure turbulence parameter $C_{n}^{2}$.

\section{CONCLUSIONS}

In this work the weak turbulence atmosphere simulator system comprising two SLMs has been described and evaluated by numerical simulations. It has been proven that it can be used for the simulation of the beam propagation over a wide interval of distances. The comparison between the theoretical and simulated results shows that the system is able to accurately reproduce the on-axis SI of a Gaussian beam propagated in diverse weak turbulence atmospheric conditions. The values of the beam spreading and beam wander are smaller than expected. This could be overcome considering other phase screen generation methods such as Zernike polynomials, among others. The considered system allows changing in a programmable way the atmospheric conditions and the propagation distances without any device displacements. We believe that it has a great potential to analyze the propagation of different types of beams and to test recently proposed FSOC protocols based on application of beams with orbital angular momentum.

\section{ACKNOWLEDGMENTS}

Spanish Ministerio de Economía y Competitividad is acknowledged for funding the project TEC2014-57394-P. C. Rickenstorff gratefully acknowledges the CONACyT postdoctoral grant 234821 . 


\section{REFERENCES}

1. L. C. Andrews and R. L. Phillips, Laser beam propagation through random media, Press Monographs, SPIE Press, 2005.

2. J. D. Schmidt, Numerical Simulation of Optical Wave Propagation with Examples in MATLAB, Press monograph, SPIE, 2010.

3. S. V. Mantravadi, T. A. Rhoadarmer, and R. S. Glas, "Simple laboratory system for generating well-controlled atmospheric-like turbulence," in Advanced wavefront control: Methods, devices and applications II, J. D. Gonglewsky, ed., Proc. SPIE 5553, pp. 290-300, 2004.

4. C. Rickenstorff, J. A. Rodrigo, and T. Alieva, "Programmable simulator for beam propagation in turbulent atmosphere," Submitted to Opt. Express, 2016.

5. H. Ren and S.-T. Wu, "Variable-focus liquid lens," Opt. Express 15, pp. 5931-5936, 2007.

6. A. Belmonte, "Feasibility study for the simulation of beam propagation: consideration of coherent lidar performance," Appl. Opt. 39, pp. 5426-5445, 2000.

7. J. Recolons and F. Dios, "Accurate calculation of phase screens for the modelling of laser beam propagation through atmospheric turbulence," in Atmospheric Optical Modeling: Measurement and simulation, S. M. Doss-Hammel, ed., Proc. SPIE 5891, pp. 1-12, 2005. 